\title{
Energy and pitch-angle dispersions of LLBL/cusp ions seen at middle altitudes: predictions by the open magnetosphere model
}

\author{
M. Lockwood \\ Rutherford Appleton Laboratory, Chilton, Didcot, OX11 0QX, UK \\ e-mail: mike@eiscat.ag.rl.ac.uk
}

Received: 10 March 1997 / Revised: 24 June 1997 / Accepted: 25 June 1997

\begin{abstract}
Numerical simulations are presented of the ion distribution functions seen by middle-altitude spacecraft in the low-latitude boundary layer (LLBL) and cusp regions when reconnection is, or has recently been, taking place at the equatorial magnetopause. From the evolution of the distribution function with time elapsed since the field line was opened, both the observed energy/observation-time and pitch-angle/energy dispersions are well reproduced. Distribution functions showing a mixture of magnetosheath and magnetospheric ions, often thought to be a signature of the LLBL, are found on newly opened field lines as a natural consequence of the magnetopause effects on the ions and their flight times. In addition, it is shown that the extent of the source region of the magnetosheath ions that are detected by a satellite is a function of the sensitivity of the ion instrument. If the instrument one-count level is high (and/or solar-wind densities are low), the cusp ion precipitation detected comes from a localised region of the mid-latitude magnetopause (around the magnetic cusp), even though the reconnection takes place at the equatorial magnetopause. However, if the instrument sensitivity is high enough, then ions injected from a large segment of the dayside magnetosphere (in the relevant hemisphere) will be detected in the cusp. Ion precipitation classed as LLBL is shown to arise from the lowlatitude magnetopause, irrespective of the instrument sensitivity. Adoption of threshold flux definitions has the same effect as instrument sensitivity in artificially restricting the apparent source region.
\end{abstract}

Key words. Low-latitude boundary layer · Cusp regions $\cdot$ Open magnetosphere model $\cdot$ Mid-altitudes

\section{Introduction}

Recently. two complementary models of the precipitation of magnetosheath ions into the dayside auroral ionosphere have been developed and have successfully reproduced the energy-latitude dispersion plume of ions spanning the precipitation regions classed cusp, mantle and polar cap. These models are based on the open magnetosphere theory in which the plasma gains entry to the magnetosphere by flowing along newly-opened field lines produced by magnetopause reconnection. The model of Onsager et al. (1993) employs fixed, steadystate distributions of magnetic and electric field throughout the dayside magnetosphere to evaluate the trajectories of the ions. The model developed by Lockwood and Smith (1994), Lockwood (1995a) and Lockwood and Davis (1996) is based on the same general principles but is different in a number of details. One of the more fundamental differences from the Onsager et al. model is that the spatial distribution of electric and magnetic fields does not need to be specified. Instead, it is assumed that the frozen-in theorem and $\mathbf{E} \cdot \mathbf{B}=0$ apply everywhere away from the reconnection site and the precipitation is studied as a function of the time elapsed since the reconnection of the field line onto which it is frozen. This is done by solving the tangential stress balance on newly opened field lines to evaluate how they evolve over the magnetopause. A major advantage of the model by Lockwood and co-workers is that it can readily be used to study time-varying situations like pulsed reconnection: an important disadvantage is that non-ideal-MHD effects (such as gradient-B and curvature drifts of the more energetic particles) cannot easily be included.

A key part of the identification of the low-latitude boundary layer (LLBL) precipitation is the presence of both magnetosheath-like and magnetosphere-like ion populations. In this respect, the presence of energetic magnetosphere-like ions, at energies above those of the ions from the magnetosheath, is a crucial defining feature of the LLBL (Woch and Lundin, 1993) and is usually interpreted as showing that the LLBL field lines are closed. However, Lockwood (1997) has recently argued that the open magnetosphere theory provides an explanation not only of the LLBL ion precipitation, but 
also of any dayside boundary plasma sheet (BPS), with both being on open field lines. The open-closed field line boundary is inferred to be equatorwards of the BPS (or void if fluxes are low or difficult to classify), close to the poleward edge of the central plasma sheet (CPS). The locations where all these classes of precipitation are typically found have been surveyed statistically by Newell and Meng (1992).

The velocity filter effect on injected magnetosheath ions was first discussed by Rosenbauer et al. (1975). These authors considered the trajectories of ions of different energies and pitch angles from a single point on the magnetopause in the presence of the large-scale dawn-to-dusk electric field which gives convection of plasma and magnetic flux from the dayside magnetosphere into the tail lobe. In particular, they provided an explanation of upgoing magnetosheath ions, seen by mid-altitude satellites in the mantle region of the magnetosphere, in terms of ions entering through the cusp region and mirroring at low altitudes in the converging geomagnetic field lines. The lower-energy ions are swept further towards the tail lobe by the fieldperpendicular convection because they have lower fieldaligned velocities and thus longer flight times (from the magnetopause down to the mirror point and then back up to the satellite). There are two competing pitch-angle effects on the flight time: higher pitch-angle ions have lower field-parallel velocities than more field-aligned ions of the same energy; however, the mirror point for smaller pitch angles is further below the satellite, making the path travelled longer.

Reiff et al. (1977) used the velocity filter concept to show that the energy-latitude dispersion of magnetosheath ions seen at low altitudes in the topside ionosphere was also due to the effect of ion flight times in a convecting magnetosphere. Hill and Reiff (1977) also showed that the ion fluxes at the high-energy end of this dispersion ramp were at higher energies than in the magnetosheath, providing evidence that they were associated with the accelerated ion flows seen at the dayside magnetopause (see review by Cowley, 1982). The highest-energy ions are found at the equatorward edge of the dispersion ramp for southward IMF, but at the poleward edge for northward IMF (Woch and Lundin, 1992).

The fact that the velocity filter effect is seen in the ion dispersion is consistent with the ions, in the main, undergoing adiabatic, scatter-free motion from the magnetopause to the satellite. This being the case, an ion's magnetic moment is conserved so that its pitch angles at its magnetopause source, at a distance $z$ along the field line from the magnetopause, and at the satellite (respectively, $a_{m}, a(z)$ and $a_{s}$ ) are related by:

$\sin ^{2} a_{m} / B_{m}=\sin ^{2} a(z) / B(z)=\sin ^{2} a_{s} / B_{s}$,

where $B_{m}, B(z)$ and $B_{s}$ are the corresponding magnetic field intensities at these three locations. Using order-ofmagnitude estimates of $B_{s}=5 \times 10^{-5} \mathrm{~T}$ (for a lowaltitude satellite, geocentric distance, $r \sim 1 \mathrm{R}_{\mathrm{E}}$ ) and $B_{m}=50 \mathrm{nT}$, and noting that ions with mirror points at or below the satellite have $a_{s}<90^{\circ}$, Eq. (1) shows that only the ions close to field-aligned at the magnetopause $\left(a_{m}<2^{\circ}\right)$ will reach satellites in the topside ionosphere.

However, at higher altitudes (and we here use a typical $r$ of $\left.4 \mathrm{R}_{\mathrm{E}}\right)$, the lower $B_{s}(\sim 200 \mathrm{nT})$ means that a larger angular slice of the distribution function at the dayside magnetopause $\left(a_{m}\right.$ up to about $30^{\circ}$ ) can be detected. The ion velocity along the field line is $(2 E / m)^{1 / 2}$ $\times \cos a(z)$, where $E$ is the (conserved) ion energy and $m$ is its mass. From Eq. (1) the time of flight $T$, along the field-aligned distance of $s$ from the magnetopause to the satellite, is therefore (Burch et al., 1986):

$T=(m / 2 E)^{1 / 2} \int_{o}^{s}\left\{1-\sin ^{2} a_{s} B(z) / B_{s}\right\}^{-1 / 2} \mathrm{~d} z$.

Note that in general, the solution of Eq. (2) requires knowledge of the field $B(z)$ at all $z$ from the magnetopause between zero and $s$ : however, this is not true in the special case of purely field-aligned ions for which $a_{s}$ is zero, and Eq. (2) reduces to $T=s(m / 2 E)^{1 / 2}$. Equation (2) shows that ions observed at larger pitch angles, $a_{s}$, have a longer time-of-flight at a given energy; conversely, for ions to have the same time of flight, higher energies are required at larger pitch angles. If we consider a point magnetopause source, the ions which are swept the same amount downstream by the convection electric field will all share the same flight time, $T$, and so those seen together at the satellite must have higher energies $E$ at higher pitch angles, $a_{s}$. On an ion spectrogram, in which ion flux is contoured as a function of energy and observation time, the ions will form a characteristic V-shape as the satellite spins and repetitively samples the full range of $a_{s}$. These "ion Vs" are observed by mid-altitude satellites in the LLBL/cusp region (e.g. Burch et al., 1982, 1985; Woch and Lundin, 1992; Kremser et al., 1995). This has therefore been interpreted as showing that the LLBL/ cusp ions come from a relatively narrow source region. A spread in source locations would allow a range of $T$ to be sampled simultaneously and so introduce a range of ion energies at any one pitch angle (which is equivalent to a spread of pitch angles at any one energy), i.e. it gives width to the ion Vs. From these ideas, Menietti and Burch (1988) used the width of the ion Vs to deduce that the source region was only about $1 \mathrm{R}_{\mathrm{E}}$ in extent.

On the other hand, Lockwood and Smith (1993) have argued that the spread of the ion energies seen in cusp at low altitudes reveals that the magnetopause source region of these magnetosheath ions must be of order 10 $20 \mathrm{R}_{\mathrm{E}}$ in extent. This is consistent with the open magnetosphere theory in which plasma streams continuously across the magnetopause at all times for which the field line is open. At times when the field line threads the dayside magnetopause, ions crossing the boundary are accelerated such that they acquire large field-aligned velocities towards the Earth as the field lines contract Earthwards. The contrasts with later times, when the field lines thread the boundary in the mantle region (the magnetopause edge of the tail lobe) where the ions 
crossing the boundary are decelerated and flow towards the tail as the field lines are stretched in the tail lobe [see discussion by Cowley et al. (1991) and Lockwood $(1995 \mathrm{a}, \mathrm{b})]$. Thus, at larger elapsed times since reconnection, most of the injected sheath ions do not precipitate down to the inner magnetosphere (and so, in the main, they reach neither low nor middle altitudes); rather, they mainly flow antisunwards into the tail lobe. In addition, the density and temperature of the source magnetosheath population is highest on the dayside, but decreases with distance away from the nose of the magnetosphere (Spreiter et al., 1966). As a result, the high-flux cusp precipitation evolves into the lower-flux, lower-energy mantle precipitation and, later, into the almost non-existent polar-cap ion precipitation. These effects mean that, although the ions continue to flow across the magnetopause as the newly opened field line evolves into the tail lobe, they cease to be a source of high fluxes in the inner magnetosphere. These effects can act to restrict the effective source region for the ions in the ionospheric cusp region to $10-20 R_{E}$, though they would not be consistent with the smaller $\left(\sim 1 R_{E}\right)$ extents derived from the ion Vs.

In this paper, we investigate whether the open magnetosphere theory is indeed consistent with the observed ion Vs and discuss the size of the source region. In Sect. 2 we extend the modelling of Lockwood (1995a) to cover all pitch angles sampled by satellites at mid-altitudes. In addition, we include the acceleration of magnetospheric ions at the magnetopause, via the mechanism proposed by Lockwood et al. (1996). The results are discussed in Sect. 3 and compared with observations. In Sect. 4 we show that the geometric factor and sensitivity of the ion detector determines how extensive the source region is inferred to be and also discuss the role of flux thresholds employed in definitions of the precipitation class.

\section{The model}

The model used is a development of that described and employed by Lockwood (1995a) to investigate the dispersion of injected sheath ions in the (open) LLBL/ cusp/mantle/polar-cap dispersion plume. This model was developed from the theory presented by Lockwood and Smith (1994) and has been used by Lockwood and Davis (1996) successfully to predict the observed ion precipitation signatures (in spectrogram format) for various low-altitude satellite passes with pulsed magnetopause reconnection. The model combines five key elements: (1) gas dynamic predictions of the morphology of the magnetosheath near the magnetopause boundary (Spreiter et al., 1966); (2) the method for determining the evolution of newly opened field lines over the magnetopause of Cowley and Owen (1989); (3) the theory of the interactions of ions with the magnetopause current sheet by Cowley (1982), recently extended to cover another magnetopause Alfvén wave by Lockwood et al. (1996); (4) the velocity filter effect of ion flight times, originally invoked by Rosenbauer et al. (1975); and (5)
Liouville's theorem of the conservation of phase space density for a dynamical processes (e.g. Elliott, 1993).

The starting point of the model is the method to compute how newly opened field lines evolve over the dayside magnetopause, away from the reconnection site, using the stress-balance concepts introduced by Cowley and Owen (1989). This is achieved using a gas-dynamic model of the boundary-tangential magnetosheath bulk flow over the boundary (Spreiter et al., 1966) and an input draped magnetosheath field orientation (as a function of position), as specified by Lockwood (1995a). (Note that this draped sheath field determines the spatial distribution of the boundary-normal field, $B_{n}$, at the magnetopause, which is therefore effectively an input to the model and not self-consistently computed). Application of the algorithm of Cowley and Owen (1989) yields not only the location where each newly opened field line threads the boundary (here referred to generically as the point $P_{n}$ ) as a function of time elapsed since reconnection, but also gives the field-line speed over the boundary (the de Hoffman-Teller velocity) at each $\mathrm{P}_{\mathrm{n}}$ (de Hoffman and Teller, 1950). We define the distance along the magnetopause (along the locus of the points where the convecting field line threads the boundary) from the reconnection site to the point $\mathrm{P}_{\mathrm{n}}$ to be $d_{n}$ and the time taken for the open field line to reach $\mathrm{P}_{\mathrm{n}}$ to be $t_{n}$. The Spreiter et al. gas-dynamic model is used to give the ion gas density and temperature at each $\mathrm{P}_{\mathrm{n}}$, and the theory of Cowley (1982) is used with the de HoffmanTeller velocity to compute the distribution function of the ions injected by flowing along the newly opened field lines across the magnetopause at each $\mathrm{P}_{\mathrm{n}}$. This theory of the ion acceleration is a vital part of both this model and that by Onsager et al. (1993): its application and its verification in ion observations from the magnetopause have been discussed by Lockwood (1995a). In order to apply the theory, the angle $\phi\left(t_{n}\right)$ that the magnetospheric part of the open field line makes with the boundary must be known. This was determined from the boundary normal field at $\mathrm{P}_{\mathrm{n}}, B_{n}$ (as just discussed), and the magnitude of the interior field $B_{s p}$, taken from a Tsyganenko T87 model in a way described in the following $\left(\phi=\sin ^{-1}\left[B_{n} / B_{s p}\right]\right)$.

The next element of the model is to compute the time of flight, $T$, of the ions from each $\mathrm{P}_{\mathrm{n}}$ to the altitude of the satellite (in this paper we consider a satellite at a geocentric distance of $r=4 \mathrm{R}_{\mathrm{E}}$ ). In the previous work, this was restricted to field-parallel ions for ease of computation (one major complication introduced in the following is to consider ions at other pitch angles): to compute $T$, Lockwood (1995a) employed the Tsyganenko T87 magnetic field model to give an estimate of the field-aligned distance $s$ from each $\mathrm{P}_{\mathrm{n}}$ to the altitude of the satellite. The T87 model contains no systematic open flux and thus field lines do not thread the magnetopause in the way required to define the $\mathrm{P}_{\mathrm{n}}$. In order to simulate the open magnetosphere, an artificial magnetopause was introduced just inside the main magnetopause current sheet implied by the model. This gave not only an estimate the required distance $s$, but also the estimate of the field strength just inside the boundary, $B_{s p}$, which 
was used to compute the angle $\phi$. With knowledge of their time of flight we can, for every time elapsed since reconnection, evaluate when and where any ions observed by the satellite were injected across the magnetopause. We here use the same notation as Lockwood (1995a), in which the time elapsed since reconnection is $t_{s}-t_{o}\left(t_{s}\right.$ is the time of observation at the satellite and $t_{o}$ is the time that the field line was opened). The point of entry of every particle is known because

$t_{s}=t_{o}+t_{n}+T$,

and so for a known $\left(t_{s}-t_{o}\right)$ and $T$, the time $t_{n}$ can be evaluated and, as already described, $d_{n}$ is known. From the distribution functions computed by the Cowley theory at each $\mathrm{P}_{\mathrm{n}}$, we know the phase space density of the ions of the energy $E$ corresponding to the flight time $T$. Because the ion motion considered is adiabatic $(\mathbf{E} \cdot \mathbf{B}=0$ in ideal MHD so field-parallel electric fields are neglected) and scatter free, both the ion energy and, by Liouville's theorem, the phase space density are conserved in travelling from $\mathrm{P}_{\mathrm{n}}$ to the satellite. Thus the distribution function $f\left(E, a_{s}\right)$ of the ions at the satellite can be computed, as a function of time elapsed since reconnection $\left(t_{s}-t_{o}\right)$. The location of the source point on the magnetopause $\mathrm{P}_{\mathrm{n}}$ (quantified by the distance $d_{n}$ ) of the ions seen at any one position in phase space $\left(E, a_{s}\right)$ at that $\left(t_{s}-t_{o}\right)$ is also known.

As already mentioned, we here extend the modelling of Lockwood (1995a) to allow for pitch angles other than zero. Equation (2) shows that in order to evaluate the time of flight $T$ for a given ion energy $E$ we need not only the distance $s$, but also the variation of the magnetic field strength $B$ along the field line along the distance $s$. This is also taken from the Tsyganenko T87 magnetic field model.

The model used here also includes the effect of acceleration of magnetospheric ions by reflection off both of the two Alfvén waves launched by the reconnection site. This concept is a generalisation of the Cowley (1982) theory and was used by Lockwood et al. (1996) to model the highest-energy ions of an observed ion dispersion plume reported by Newell et al. (1991a). A second observed example, reported by Moen et al. (1996), was also successfully explained using this theory (Lockwood and Moen, 1996). The basic concept is illustrated in Fig. 1. This schematic shows three newly opened field lines emanating from a reconnection site, $\mathrm{X}$; the field line passing through $\mathrm{X}$ makes up the two magnetic separatrices (s) and is for a $\left(t_{s}-t_{o}\right)$ of zero; the other two field lines are for larger $\left(t_{s}-t_{o}\right)$. The reconnection site, X, launches two Alfvén waves (also called intermediate mode or rotational discontinuity, RD) shown by the dashed lines: the interior wave, $i$, stands in the inflow to the reconnecting boundary on the magnetospheric side, the exterior wave, e, stands in the inflow from the magnetosheath side. In the sense that the majority of the field rotation takes place at it, e is the magnetopause. In this simplified view of the reconnecting magnetopause, all of the field rotation takes place at these two Alfvén waves and, because the Alfvén speed is much greater on the magnetospheric side of the boun-

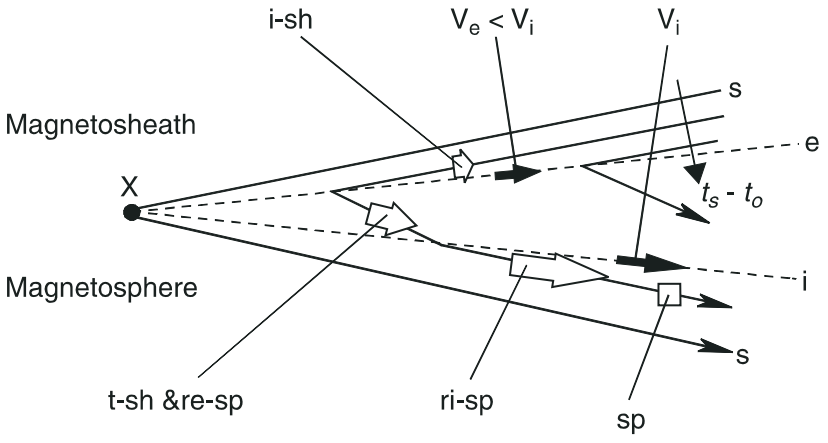

Fig. 1. Schematic of the open LLBL emanating from a magnetopause reconnection site, $\mathrm{X}$. The magnetic separatrices s are the locations of the field lines at time elapsed since reconnection $\left(t_{s}-t_{o}\right)=0$ : newly opened field lines are also shown for two later $\left(t_{s}-t_{o}\right)$. The exterior and interior Alfvén waves (launched by the reconnection site into the magnetosheath and magnetosphere inflow regions) are e and i, along which the kinks in the newly opened field lines evolve at speeds $V_{e}$ and $V_{i}$. The bulk motions (in the Earth's frame of reference) of various ion populations discussed in the text are shown with broad arrows.

dary (owing to the lower plasma density), the field kink at the interior wave moves at a speed $V_{i}$ which is considerably greater than that of the kink at the exterior wave, $V_{e}$. It should be noted that MHD theories suggest a more complex structure than is proposed here (e.g. Biernat et al., 1989), including a fast wave, an intermediate shock and a slow expansion wave on the magnetosheath side of the reconnection layer, and a fast wave, Alfvén wave and slow shock on the magnetospheric side: the two sides being separated by a contact discontinuity. Although such structure is reproduced in MHD simulations, it is not found in hybrid simulations, which yield the simpler picture of a mixing of the two plasmas in an open LLBL which is bounded by two Alfvén waves (Lin and Lee, 1993). The attraction of these hybrid simulations (for the work presented here) is that they also reproduce the ion distribution functions seen near the magnetopause, as predicted by Cowley (1982) and observed by Gosling et al. (1990), Fuselier et al. (1991) and Smith and Rodgers (1991). An important part of Cowley's predictions is that ions incident on an $\mathrm{RD}$ are either transmitted through it or reflected off it. The hybrid simulations by Chapman and Moukis (1996) show that this occurs because ions fall into a potential well at the centre of the RD and emerge (with the same energy and pitch angle) on one or other side, depending on their initial gyrophase. Thus a fraction $r$ of incident ions at a certain point in $\left(\mathrm{V}_{\perp}, \mathrm{V}_{\|}\right)$velocity space are reflected while the remaining fraction $(1-r)$ are transmitted. Chapman and Moukis also show that this does not occur in ideal MHD simulations. This behaviour is almost exactly as assumed by Cowley (1982) when he made his predictions.

Figure 1 shows the bulk motion of the relevant ion populations in the frame of the Earth. The magnetosheath population adjacent to the boundary is shown flowing away from the X-line, but because its speed is considerably slower than $V_{e}$, it is effectively being overtaken by the field-line kink and will be incident 
upon the boundary e. This results in the magnetosheath plasma motion in the field line rest frame (the de Hoffman-Teller frame) being purely field aligned and towards the RD at the local Alfvén speed, $V_{A s h}$, i.e. the Whalén relation holds (Cowley and Owen, 1989). This population is therefore labelled i-sh (i for incident, sh for sheath origin). Some of this i-sh population will pass through the magnetopause to form the $\mathrm{t}$-sh population (t for transmitted) which near the X-line moves Earthwards with a bulk flow speed of about $\left(V_{e}+V_{A s h}\right)$ in the Earth's frame. Lockwood (1995a, b) has discussed how this velocity and the t-sh ion population subsequently evolves with the distance $d_{n}$. The changes occur for three reasons: firstly, the field line accelerates away from the reconnection site towards the tail lobe; secondly the ion acceleration decays as the field line straightens (specifically, the angle $\phi$ that the magnetospheric arm of the field line makes with the magnetopause increases); thirdly, the characteristics of the magnetosheath source plasma change with $d_{n}$. On the magnetospheric side of the boundary, the ion population, $\mathrm{sp}$, is at rest in the Earth's frame and thus the interior kink of the newlyopened field lines will engulf it at speed $V_{i}$, which will be the local (interior) Alfvén speed, $V_{A s p}$ (i.e, as at the exterior RD, the Whalén relation holds). On interaction with the wave $i$, any $\mathrm{sp}$ ions reflected move away from the boundary and towards Earth with bulk flow speed of about $2 V_{A s p}$ (these reflected ions are referred to ri-sp: ri for reflected off the interior wave and $\mathrm{sp}$ for magnetosphere in origin). Note however, that the ri-sp population can only be seen from the magnetospheric side of $i$, so at low altitudes they should only found equatorward of the interior wave. Most sp ions will pass through $\mathrm{i}$ and some of these will be reflected off $\mathrm{e}$ to give a population termed re-sp, which near the X-line has an Earthward bulk flow speed of about $2 V_{e}$. The re-sp population will evolve with $\left(t_{s}-t_{o}\right)$ for three reasons. The first two reasons are the same as for the t-sh population, namely the de Hoffman-Teller velocity changes as the field line accelerates, and acceleration decays as the field line straightens. The third reason is that the source $\mathrm{sp}$ ions are progressively lost by interaction with the magnetopause. Once the field line is opened a loss of $\mathrm{sp}$ ions commences, either because they are reflected off the magnetopause to become re-sp or ri-sp ions, or because they are transmitted through it into the magnetosheath. At any one $\left(t_{s}-t_{o}\right)>0$, the sp ions which remain are those which have not had time to interact with the open magnetopause: they have a halfbounce time-of-flight (from the reconnection site down to a mirror point and back up to the magnetopause) which is greater than $\left(t_{s}-t_{o}\right)$.

Applying the theory of Cowley (1982) to each of these populations, the model generates the total ion distribution functions at each $\mathrm{P}_{\mathrm{n}}$ in the open LLBL: in the example shown in Fig. $2, \mathrm{P}_{\mathrm{n}}$ is in the immediate vicinity of a subsolar reconnection site (i.e. $d_{n}$ and $t_{n}$ are nearly zero in this example). These plots show the phase space density, $f$, colour coded as a function of the fieldperpendicular and field-parallel ion velocities (in the Earth's frame of reference and positive towards the
Earth). The upper plot is for the part of the open LLBL that is between the interior wave $\mathrm{i}$ and the ion edge (which is closer to the separatrix $s$ in Fig. 1). On the other hand, the lower plot is for within the open LLBL and between the two waves $i$ and $e$. The input parameters to the model are derived to give the same conditions at the reconnection site (at the nose of the magnetosphere) as were deduced from observations in the low-altitude cusp region by Lockwood et al. (1994, 1996): they are listed in full in Table 1. Note that many of the parameters are not fixed by the theory or simulations discussed here (for example the reflection coefficients $r_{i}$ and $r_{e}$ ) and values used are simply those that give the best fits to the data. The ri-sp ions can only be seen in the upper plot and appear at the highest Earthward field-aligned velocities. The main peak in $f$ shows the characteristic D-shaped distribution of the $\mathrm{t}$ sh population, as predicted by Cowley (1982) and as has been observed in a number of studies (Gosling et al., 1990; Fuselier et al., 1991; Smith and Rodgers, 1991) and produced in the hybrid simulations of Lin and Lee (1993). The population centred on the origin is the initial, isotropic sp population (usually called the central plasma sheet, CPS). Figure 3 gives the spectra of the field-aligned ions $\left(a_{m}=0\right)$ of the various components of the total distribution function shown in Fig. 2a. Figure 3 is in the form of a log-log plot of the differential energy flux, $J_{E}$, as a function of the energy, $E$. The t-sh, sp, resp and ri-sp populations are labelled. Note that in this paper, we make predictions of the phase space density, $f$, the differential number flux (sometimes referred to as the intensity), $J$, and the differential energy flux, $J_{E}$ : at any one energy, $E$, these are related by:

$J_{E}=f\left(2 / m^{2}\right) E^{2}=J E$.

Figure 4a shows an example set of flight times of all the ions injected/accelerated at this $\mathrm{P}_{\mathrm{n}}$, in the immediate vicinity of the $\mathrm{X}$-line $\left(d_{n} \approx 0\right)$, to a mid-altitude satellite at a geocentric distance of $r=4 \mathrm{R}_{\mathrm{E}}$. These are computed from the magnetic field model, using Eq. (2). The time of flight, $T$, is colour coded on a logarithmic scale as a function of the field-perpendicular and field-parallel ion velocities of the ions when they reach the satellite. The flight times can be used in the way described to determine the distribution function $f\left(E, a_{s}\right)$ at a given elapsed time since reconnection $\left(t_{s}-t_{o}\right)$.

\section{Results}

\subsection{Distribution functions}

Parts b-f of Fig. 4 show a set distribution functions generated in the manner described in the previous section and using the model inputs listed in Table 1. The sequence shows how the distribution function evolves with increasing elapsed time since reconnection, $\left(t_{s}-t_{o}\right)$. From the preceding discussion of the model, it should be remembered that this evolution is caused by a convolution of three factors: the spatial variation of the ion gas in the magnetosheath, the effects of the motion of 

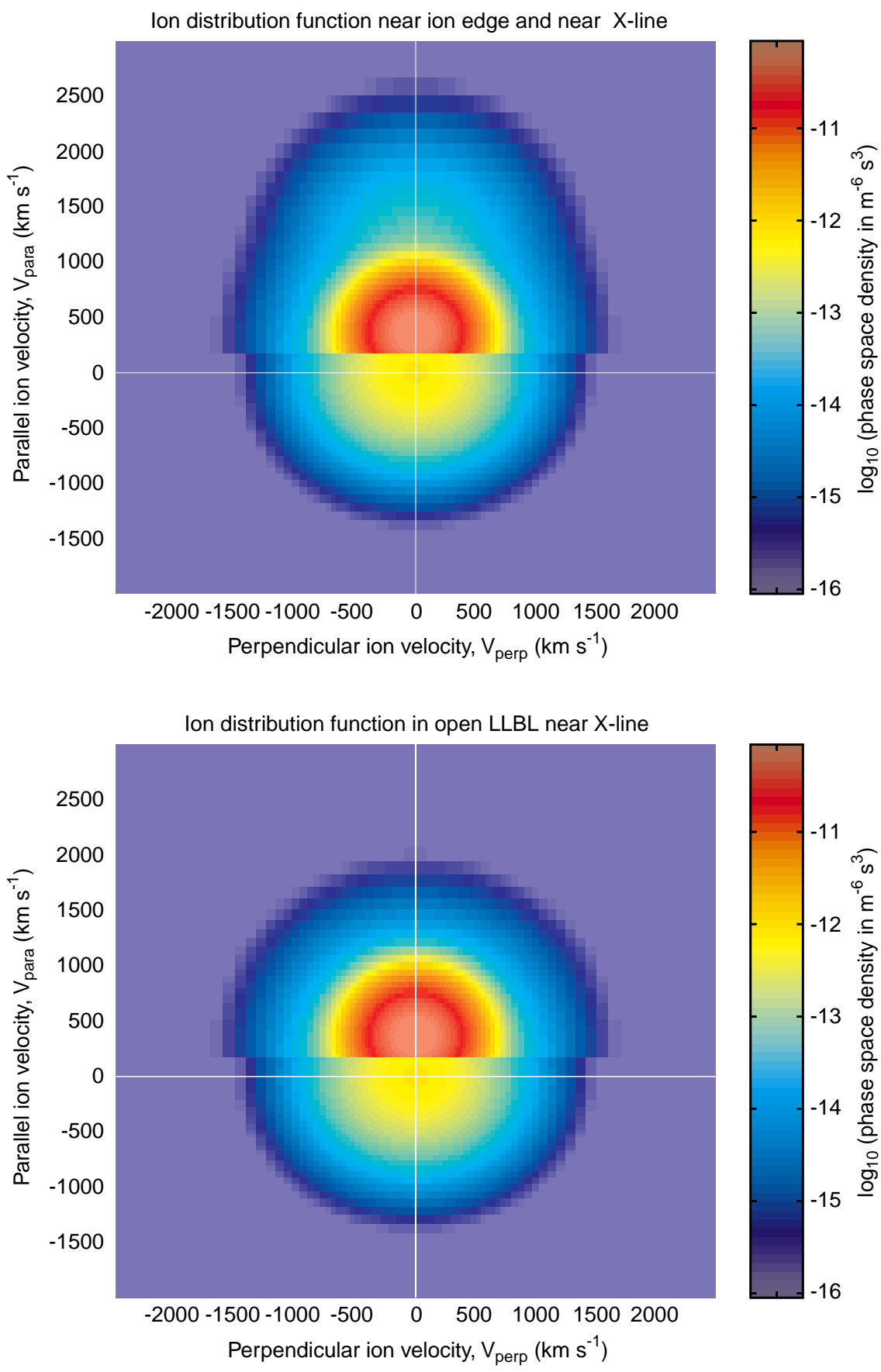

Fig. 2. Ion distribution functions in the open LLBL in the immediate vicinity of the reconnection $\mathrm{X}$-line $\left(d_{n} \approx 0, t_{n} \approx 0\right)$. The parallel velocity is positive towards the Earth and is in the Earth's frame of reference: (top) between the interior wave (i in Fig. 1) and the ion edge which is closer to the separatrix s; (bottom) between the exterior and interior waves (e and i)

the field line tailward and the time of flight of the ions from the magnetopause to the satellite.

In Fig. $4 \mathrm{~b},\left(t_{s}-t_{o}\right)=75 \mathrm{~s}$ and two ion populations can be seen. The lower-energy population is a loss-cone distribution of the sp ions (i.e. CPS) which were present on closed field lines $\left(t_{s}-t_{o}<0\right)$ and have yet to be influenced by the fact that the field line has been opened. Note that it has been assumed here that equatorial scattering has filled the loss cone corresponding to mirror points in the opposite hemisphere; however, this assumption was not necessary and a double loss cone distribution could equally well have been used. At the satellite, the loss of $\mathrm{sp}$ ions is first noted at the highest energies as the lowest flight time sp ions fail to arrive: for $\left(t_{s}-t_{o}\right)$ as low as $75 \mathrm{~s}$, only loss of ions with $T<$ $\left(t_{s}-t_{o}\right)=75 \mathrm{~s}$ could be noted and the fluxes of such ions in the sp population is negligibly small. The boundary in $\left(\mathrm{V}_{\perp}, \mathrm{V}_{\|}\right)$phase space defined by $T=\left(t_{s}-t_{o}\right)$ is here called the time-of-flight cut-off. Below this cut-off only the sp ions from the closed fieldline region can be seen, above it only the populations generated by the open magnetopause (t-sh, re-sp and ri$\mathrm{sp}$ ) can be found. For reference with Fig. 3, the lower cut-off energy of field-aligned ions is given by:

$E_{i c}\left(\alpha_{s}=O\right)=(m / 2)\left\{s_{x} /\left(t_{s}-t_{o}\right)\right\}^{2}$,

where $s_{x}$ is the distance along the field line from the $\mathrm{X}$ line to the altitude of the satellite, which is here $23.5 R_{E}$. 
Table 1. Input parameters to the model

\begin{tabular}{|c|c|}
\hline parameter & value \\
\hline solar-wind density, $N_{s w}$ & $5.67 \times 10^{6} \mathrm{~m}^{-3}$ \\
\hline solar-wind temperature, $T_{s w}$ & $2.09 \times 10^{5} \mathrm{~K}$ \\
\hline solar-wind Mach number, $M_{s w}$ & 8 \\
\hline polytropic index, $\gamma$ & $5 / 3$ \\
\hline $\begin{array}{l}\text { stand-off distance of magnetospheric } \\
\text { nose, } d_{m}\end{array}$ & $12 \mathrm{R}_{\mathrm{E}}$ \\
\hline $\begin{array}{l}\operatorname{GSE}(X, Y, Z) \text { co-ordinates of } \\
\text { reconnection site }\end{array}$ & $\left(12 \mathrm{R}_{\mathrm{E}}, 0,0\right)$ \\
\hline $\begin{array}{l}\text { field-aligned distance from reconnection } \\
\text { site to satellite height, } s_{x}\end{array}$ & $23.5 \mathrm{R}_{\mathrm{E}}$ \\
\hline mean magnetospheric CPS ( $\mathrm{sp}$ ) ion mass & 1 a.m.u. \\
\hline mean solar wind ion mass & 1 a.m.u. \\
\hline solar-wind speed, $V_{s w}$ & $500 \mathrm{~km} \mathrm{~s}^{-1}$ \\
\hline density of magnetospheric CPS ions, $N_{s p}$ & $0.4 \times 10^{6} \mathrm{~m}^{-3}$ \\
\hline temperature of magnetospheric CPS ions, $T_{s p}$ & $1.4 \times 10^{7} \mathrm{~K}$ \\
\hline Alfvén speed at interior $\mathrm{RD}, V_{A s p}$ & $600 \mathrm{~km} \mathrm{~s}^{-1}$ \\
\hline Alfvén speed at exterior RD, $V_{A s h}$ & $170 \mathrm{~km} \mathrm{~s}^{-1}$ \\
\hline $\begin{array}{l}\text { fraction of CPS ions reflected off the } \\
\text { interior RD, } r_{i}\end{array}$ & 0.1 \\
\hline $\begin{array}{l}\text { fraction of sheath ions transmitted } \\
\text { through the exterior RD, } t_{e}\end{array}$ & 0.5 \\
\hline $\begin{array}{l}\text { fraction of sp ions reflected off the } \\
\text { exterior RD, } r_{e}\end{array}$ & 0.4 \\
\hline $\begin{array}{l}\text { heating factor of sp ions at the } \\
\text { interior RD, } h_{i}\end{array}$ & 1.3 \\
\hline $\begin{array}{l}\text { heating factor of i-sh and sp ions } \\
\text { at the exterior RD, } h_{e}\end{array}$ & 1.5 \\
\hline
\end{tabular}

For Fig. 4b, $E_{i c}$ is $2.08 \times 10^{4} \mathrm{eV}$ (in Fig. $3, \log _{10}\left(E_{i c}\right)$ is 4.32).

In addition to the sp population in Fig. $4 b$, a second higher-energy population forming a "halo" has appeared which is not seen on closed field lines. These are only seen above the time-of-flight cut-off and Fig. 3 shows that for $\log _{10}\left(E_{i c}\right)>4.32$ the dominant ions are ri-sp. Thus the halo is caused by ri-sp ions reaching the

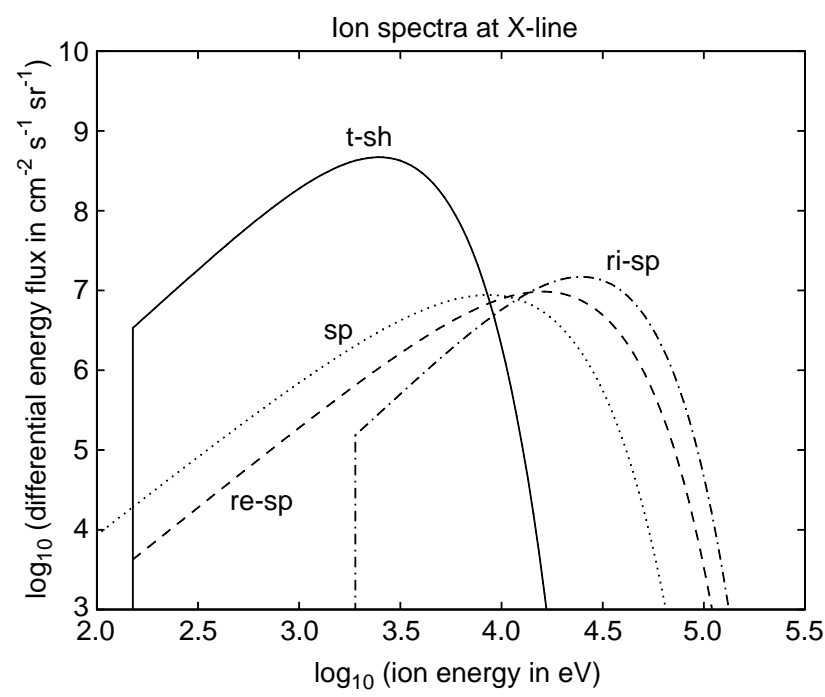

Fig. 3. The spectra of field-aligned ions (pitch angle, $a_{m}=0$ ) in the LLBL at the magnetopause, corresponding to the top part of Fig. 2. The various component ion populations, t-sh, sp, ri-sp and re-sp are discussed in the text. This plot gives the differential energy flux $J_{E}$ as a function of energy $E$ on a $\log -\log$ scale satellite. Note that somewhat lower energies have reached the satellite at $a_{s}=0$ than for larger pitch angles, in accordance with Eq. (2). This population has developed further in Fig. $4 \mathrm{c}$ which is for $\left(t_{s}-t_{o}\right)$ $=100 \mathrm{~s}\left(E_{i c}=1.17 \times 10^{4} \mathrm{eV} ; \log _{10}\left(E_{i c}\right)=4.07\right)$, as lower energy ri-sp and re-sp ions have had time to reach the satellite. At a $\left(t_{s}-t_{o}\right)$ shortly after this, the (apparent) gap between the t-sh and re-sp/ri-sp populations disappears. Lockwood (1997) argues that the ion populations in Fig. $4 b$ and c would qualify them as a BPS precipitation.

Figure $4 \mathrm{~d}$ is for $\left(t_{s}-t_{o}\right)=200 \mathrm{~s}\left(E_{i c}=2.93 \times 10^{3}\right.$ $\left.\mathrm{eV} ; \log _{10}\left(E_{i c}\right)=3.47\right)$, when substantial changes have taken place. Firstly, the interior Alfvén wave has passed over the satellite, which can therefore no longer detect any ri-sp ions. This means that the re-sp population is revealed at the highest energies. In addition, the time-offlight cut-off has moved down to still lower values and Fig. 3 shows that for this $E_{i c}$, t-sh ions will dominate just above the time-of-flight cut-off (giving phase-space densities of over $10^{11} \mathrm{~m}^{-6} \mathrm{~s}^{3}$, shown in red in Fig. 4d). The sp ions at energies above the time-of-flight cut-off (ions with $T>t_{s}-t_{o}$ ) have been lost, but this loss is masked by the presence of the t-sh and re-sp ions. The dashed line in Fig. 5 shows the spectrum of field-aligned ions at the satellite at this $\left(t_{s}-t_{o}\right)$, shown here as the differential number flux, $J$, and as a function of energy on a log-log scale. The presence of the re-sp ions at high energies means that the satellite observing a field line of this $\left(t_{s}-t_{o}\right)$ would classed as being in the LLBL (Woch and Lundin, 1993).

At $\left(t_{s}-t_{o}\right)=300 \mathrm{~s}$ (Fig. 4e), the time-of-flight cutoff has moved to yet lower velocities $\left(E_{i c}=1.30 \times 10^{3}\right.$ $\left.\mathrm{eV} ; \log _{10}\left(E_{i c}\right)=3.11\right)$, below which sp ions are seen, but above which t-sh ions and a few remnant re-sp ions are seen. The population is now like that seen in the magnetosheath so that it is classed as cusp or cusp proper. Lastly, for $\left(t_{s}-t_{o}\right)=500 \mathrm{~s} \quad\left(E_{i c}=468 \mathrm{eV}\right.$; $\left.\log _{10}\left(E_{i c}\right)=2.67\right)$, Fig. 4f shows that the re-sp ions are almost all gone and the lower cut-off energy of the tsh population has moved to yet lower values. This precipitation is also classed as cusp. This is confirmed by the differential number flux spectrum of field-parallel ions, shown by the solid line in Fig. 5. [Compare with, for example, the cusp spectra in Figs. 1 and 2 of Woch and Lundin (1992)]. As the flux is approaching the lower limit for the cusp classification, this case is quite close to the boundary of the cusp and mantle precipitation regions (Newell et al., 1991b).

Comparison of Fig. 5 with the cusp and LLBL spectra presented in Fig. 1 of Woch and Lundin (1992) shows that the model is able to reproduce the important features of both the cusp and the LLBL precipitations. Note that the model predictions presented here differ from the observed examples at low energies $(10 \mathrm{eV}-1$ $\mathrm{keV}$ ); this is because the observed CPS population contains a low-energy ionospheric component (cf. Fig. 2 of Woch and Lundin, 1993), which is not included in Fig. 5: for simplicity, the sp population used in the model was a single Maxwellian. The model also explains the evolution of the LLBL spectrum into that of the 


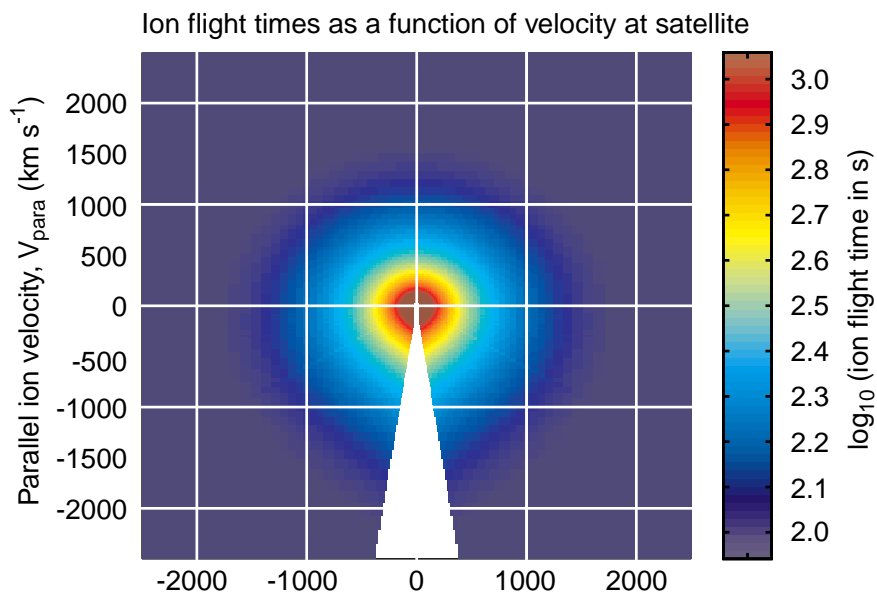

a

Perpendicular ion velocity, $\mathrm{V}_{\text {perp }}\left(\mathrm{km} \mathrm{s}^{-1}\right)$

$t_{s}-t_{o}=100 \mathrm{~s} \quad$ Ion distribution function at mid altitudes

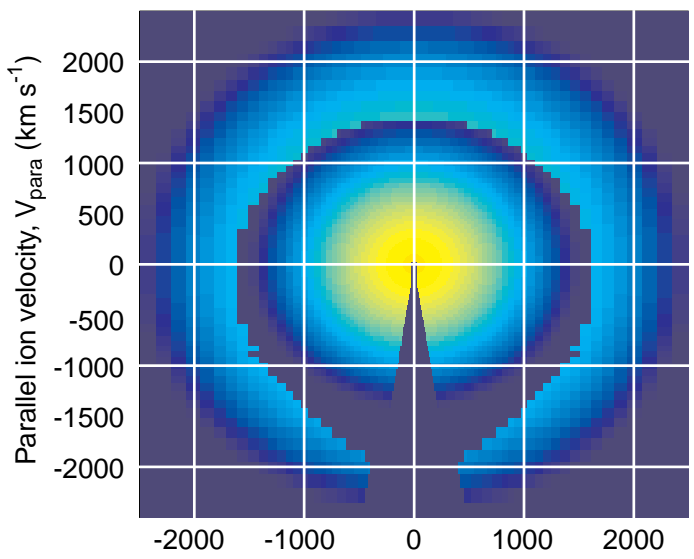

c Perpendicular ion velocity, $\mathrm{V}_{\text {perp }}\left(\mathrm{km} \mathrm{s}^{-1}\right)$

$t_{s}-t_{o}=300 \mathrm{~s} \quad$ Ion distribution function at mid altitudes

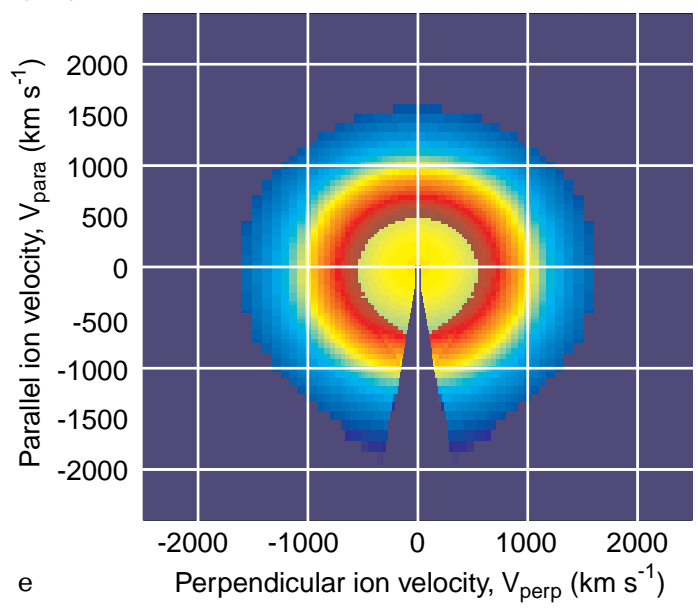

Fig. 4a. Ion flight time from a point $\mathrm{P}_{n}$ on the magnetopause to the altitude of satellite, $T$, colour coded (on a logarithmic scale) as a function of the field-parallel and field-perpendicular ion velocities at the satellite. In this example, $\mathbf{P}_{n}$ is at the (subsolar) reconnection site

cusp, for example as revealed by the sequence shown in Fig. 2 of Woch and Lundin (1992).

In cases such as that for $\left(t_{s}-t_{o}\right)=200 \mathrm{~s}$, the magnetospheric (sp) ions below the cut-off appear to belong to the same population as those magnetospheric ions that have been reflected off the exterior wave (re-sp)

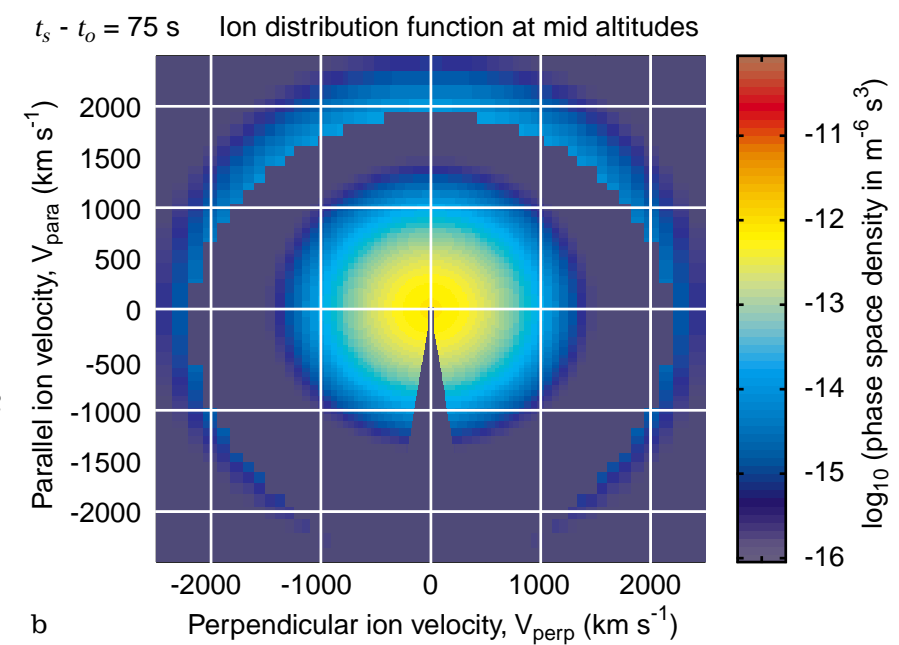

$t_{s}-t_{o}=200 \mathrm{~s} \quad$ Ion distribution function at mid altitudes

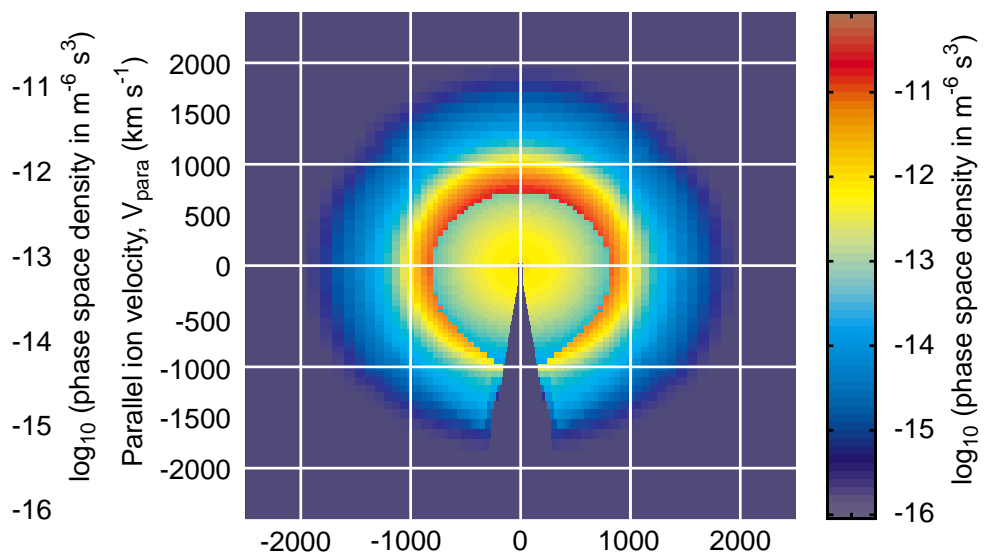

d Perpendicular ion velocity, $\mathrm{V}_{\text {perp }}\left(\mathrm{km} \mathrm{s}^{-1}\right)$

$t_{s}-t_{o}=500 \mathrm{~s} \quad$ Ion distribution function at mid altitudes

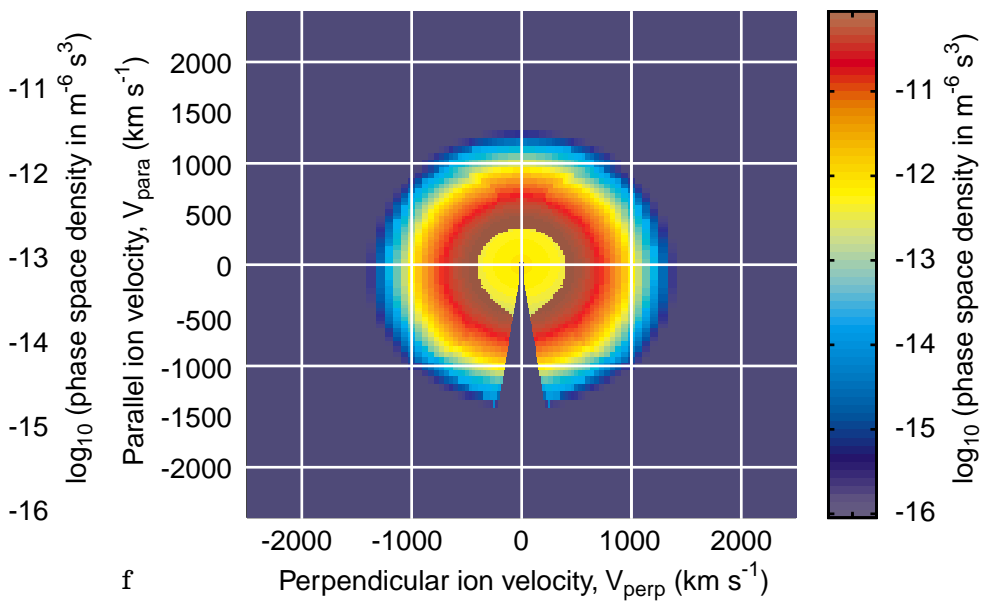

$\left(d_{n}=0, t_{n}=0\right)$. b-f Ion distribution functions at the satellite; the elapsed time since reconnection $\left(t_{s}-t_{o}\right)$ is: b $75 \mathrm{~s} ; \mathbf{c} 100 \mathrm{~s} ; \mathbf{d} 200 \mathrm{~s}$; e $300 \mathrm{~s}$; and f $500 \mathrm{~s}$

and that are seen at energies above where transmitted sheath (t-sh) ions dominate: these two populations (sp and re-sp) could therefore be fitted with a single Maxwellian (of higher temperature and density than the sp population - cf. Fig. 3). This point is demonstrated in Fig. 6, in which the $J(E)$ spectrum for 


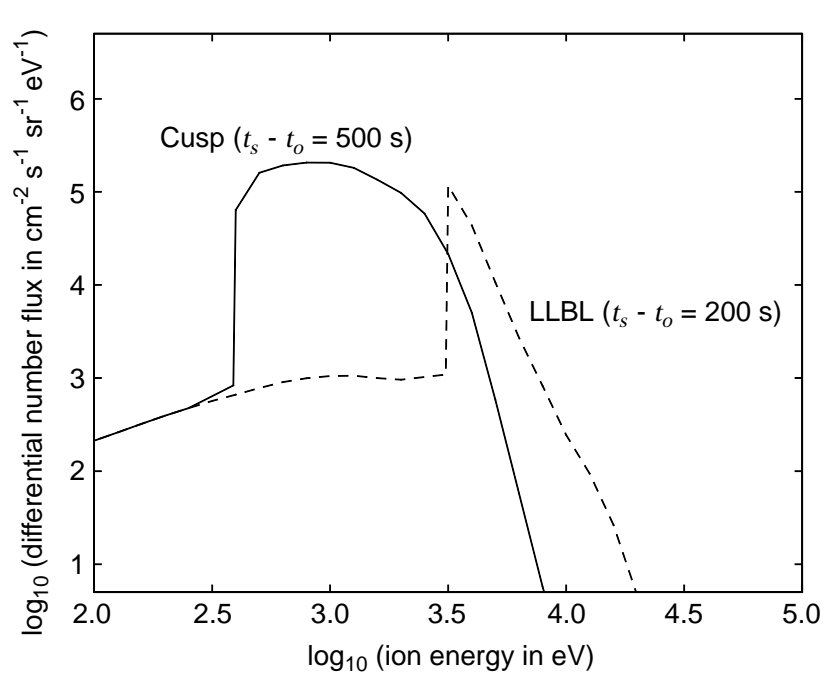

Fig. 5. Differential number flux (intensity) ion spectra at two elapsed times since reconnection. The dashed line is for $\left(t_{s}-t_{o}\right)$ of $200 \mathrm{~s}$ and is typical of spectra in the LLBL; the solid line is for $\left(t_{s}-t_{o}\right)$ of $500 \mathrm{~s}$ and is typical of spectra in the cusp. The differential number flux $J$ is plotted as a function of energy $E$ on a $\log -\log$ scale.

$\left(t_{s}-t_{o}\right)=200 \mathrm{~s}($ dashed line, as in Fig. 5) is shown with two Maxwellian spectra A and B: A is the sp population that was input into the model (with temperature $T_{s p}=1.4 \times 10^{7} \mathrm{~K}$ and density $N_{s p}=4 \times 10^{5} \mathrm{~m}^{-3}$ ) whereas $\mathrm{B}$ is a fit to the visible parts of the sp and re$\mathrm{sp}$ populations model (with temperature $T_{s p}=2.5 \times 10^{7} \mathrm{~K}$ and density, $\left.N_{s p}=8 \times 10^{5} \mathrm{~m}^{-3}\right)$. The only indication that this fit may not be valid is the fact that the observed fluxes are a bit lower at energies just below the time-of-flight cut-off, $E_{i c}$. This situation can indeed be seen in the boundary-layer spectra presented in Fig. 1 of Woch and Lundin, (1992). Note

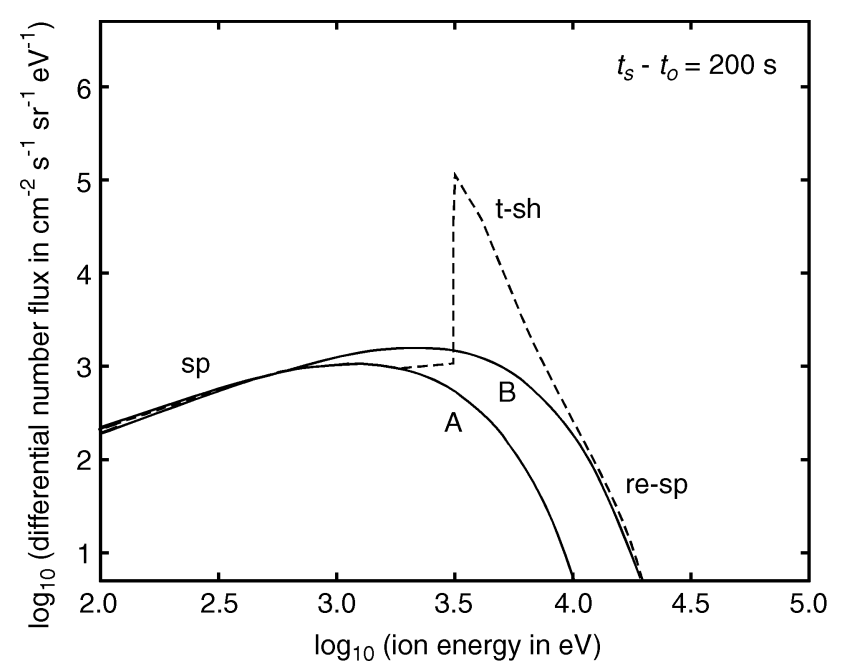

Fig. 6. The intensity spectrum for $\left(t_{s}-t_{o}\right)=200 \mathrm{~s}$, as shown in Fig. 5 (dashed line), compared with two Maxwellian sp populations: A is the input to the model with temperature $T_{s p}=1.4 \times 10^{7} \mathrm{~K}$ and density, $N_{s p}=4 \times 10^{5} \mathrm{~m}^{-3}$; B is a fit to the visible parts of the sp and re-sp populations with temperature $T_{s p}=2.5 \times 10^{7} \mathrm{~K}$ and density, $N_{s p}=8 \times 10^{5} \mathrm{~m}^{-3}$. The dominant ion population in the three parts of the total spectrum are marked (sp at low energies, t-sh at middle energies and re-sp at the high energies). that the re-sp ions can appear as a continuation of the sp ions because although only some of the sp ions are lost at the magnetopause, those that are reflected are accelerated. The slope of the sp distribution function $f(E)$ is steep at higher energies and so the rise in ion energy $E$ on reflection acts to increase $f$ and so counteracts the loss of ions by transmission through the magnetopause.

Therefore, an important point about Fig. 4d and 6 is that this distribution function [for $\left(t_{s}-t_{o}\right)=200 \mathrm{~s}$ ] appears to be a mixture of an sp population, with magnetosheath ions added, whereas, in fact, the higher energy ions are re-sp and not $\mathrm{sp}$ at all. This situation is that expected in the LLBL, with a mixture of magnetosphere-like and magnetosheath-like populations seen at the same location. The apparent presence of a full magnetospheric population is one reason why this LLBL is thought to be on closed field lines; however, the modelled distributions are on open field lines $\left(t_{s}-t_{o}>0\right)$, and the population which mimics the CPS is in fact made up of the remnant sp population at low energies (ions with $T<t_{s}-t_{o}$ ) with the re-sp population at higher energies. These effects therefore offer a good explanation of sheath plasma injected onto seemingly closed magnetospheric field lines in the LLBL: they are on field lines which have been open for a time of order $200 \mathrm{~s}$.

\subsection{Pitch-angle dispersion}

The distribution functions like those shown in Fig. 4 can be re-plotted in a pitch angle-energy format, as often used for satellite data. This is done here in Fig. 7. The distribution function has been converted to differential energy flux using Eq. 4 because either this or count rates are usually plotted (count rates being proportional to differential energy flux for a differential ion instrument). In Fig. 7, the spin-angle distributions are plotted for eight different $\left(t_{s}-t_{o}\right)$ values, as given at the top of each panel. The differential energy flux $J_{E}$ is colour coded on a logarithmic scale as a function of the logarithm of the ion energy, $E$, and the pitch angle $a_{s}$ : in each panel the pitch angle varies linearly from $a_{s}=180^{\circ}$ on the left, to $a_{s}=0$ (the downward field-aligned direction) at the centre and then returns linearly to $a_{s}=180^{\circ}$ on the right.

In the first panel of Fig. $7\left(t_{s}-t_{o}=40 \mathrm{~s}\right)$ we see mainly the sp population which cannot be differentiated from that on closed field lines as the ion loss is only at such high energies that the fluxes could not be detected anyway. However, ions belonging to the "halo" of ri-sp ions, as seen in Fig. 4b, could be seen at low pitch angles by detectors if their one-count level was below (an unrealistically low) $10^{-2} \mathrm{~cm}^{-2} \mathrm{~s}^{-1} \mathrm{sr}^{-1}$. This higherenergy population grows in flux and descends in minimum energy as $\left(t_{s}-t_{o}\right)$ increases giving a "bowllike" appearance on the spectrogram. By $\left(t_{s}-t_{o}\right)=200 \mathrm{~s}$, the first magnetosheath ions can be seen at the lower pitch angles and lower energies of this bowl feature, after which the characteristic V-shaped feature of the cusp precipitation becomes clear. This 


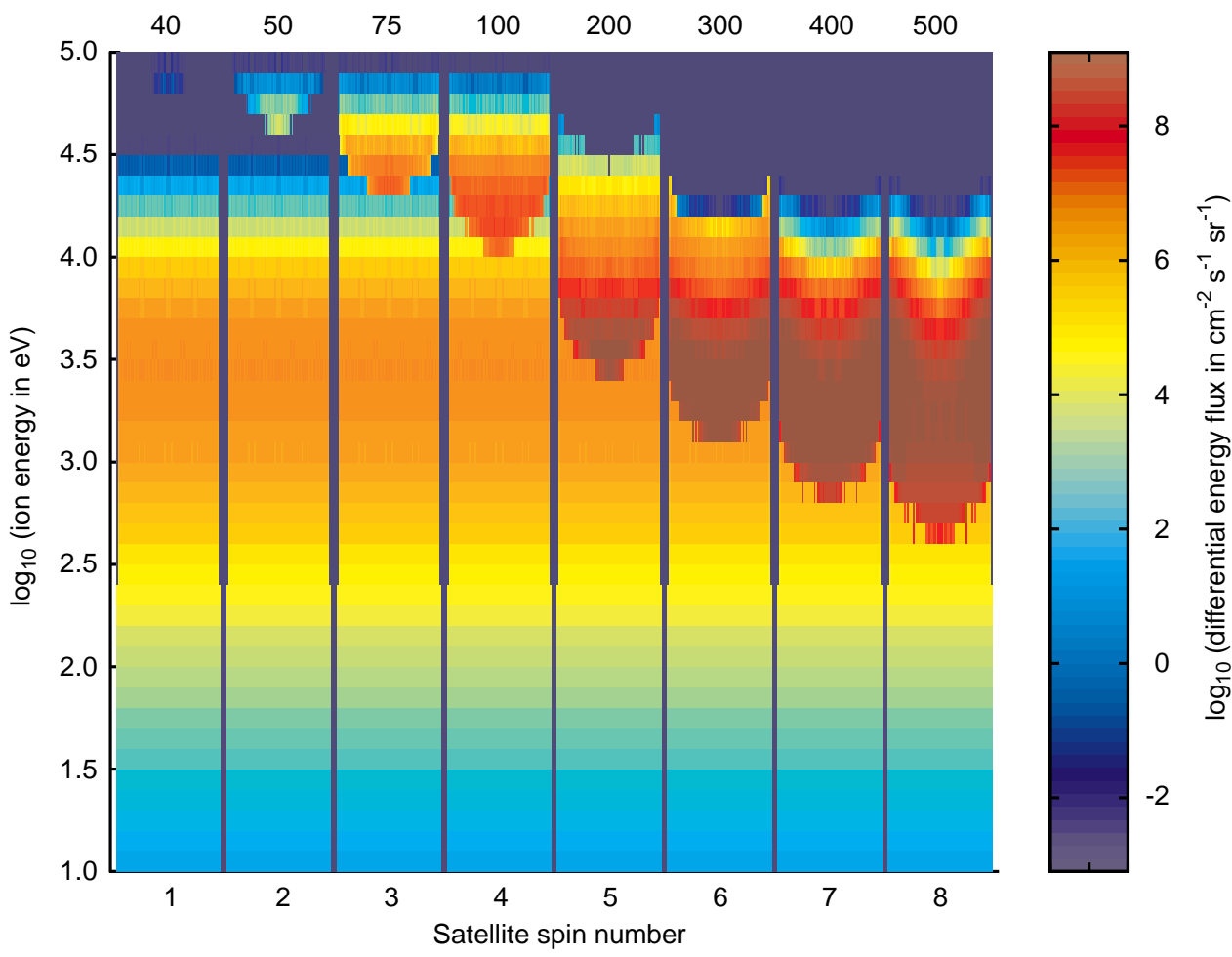

Fig. 7. Pitch-angle/energy spectra obtained by spins of a mid-altitude satellite at eight elapsed times since reconnection $\left(t_{s}-t_{o}\right)$, as given at the top of each case. The differential energy flux is colour contoured (on a $\log$ scale) as a function of pitch angle and the logarithm of energy: in each panel, the pitch angle $a_{s}$ varies from $180^{\circ}$ to 0 (downward) and back to $180^{\circ}$. development of the form from a bowl to a $\mathrm{V}$ was noted by Menietti and Burch (1988). Note that at the lower edge of the ion Vs, all ions have come from the reconnection site because they have the lowest energy and thus longest flight time $T$ at that pitch angle $a_{s}$. As a result, this edge is not dependent on the contour level, but this is certainly not true of the upper edge of the V. Thus the width of the V (in energy at any one pitch angle) depends on the sensitivity of the instrument, specifically the geometric factor and the one-count level. As a result, the extent of the source region inferred from the $\mathrm{V}$ will depend on the instrument. This point is addressed further in the next section.

Comparison of Fig. 7 with observations shows that the model reproduces well the observed ion Vs in this spectrogram format. For example, Fig. 7 can be compared with the second panel of Fig. 2 of Kremser et al. (1995). In making this comparison, it must be remembered that Fig. 7 has not been convolved with any instrument response characteristics and many of the features shown will be below the one-count level. In particular, note that the $J_{E}$ scale in Fig. 7 covers 12 decades, whereas the data presentation given by Kremser et al. (1995) covers only 3.7. For this reason, the low-flux features at the highest energies are not as clear in the data as they are in this model. Nevertheless, the data clearly reveal ions at higher energies than are seen equatorwards of the cusp on closed field lines. The bottom panel of Fig. 2 of Kremser et al: (1995) is from a high-energy-ion instrument which detects ions of energy up to about $100 \mathrm{keV}$, as in Fig. 7. These high-energy ions are observed to share the same energy/time-ofobservation dispersion ramp as the cusp ions, as is also predicted in Fig. 7. Furthermore, from the ratios of the fluxes of different species, Kremser et al. (1995) find that these higher-energy ions are of magnetospheric origin and suggest that they are generated by interaction with the magnetopause. This is confirmed to be the mechanism in the modelling presented here.

Looking closely at the spin-angle distribution for $\left(t_{s}-t_{o}\right)=500 \mathrm{~s}$, it can be seen that a minimum is starting to form at zero pitch angle, with peaks at larger values. This is also seen in the data presented by Kremser et al. (1995) and represents the evolution towards upgoing, mirrored mantle ions, as discussed by Rosenbauer et al. (1975).

Note that in Fig. 4, 5 and 7, the magnetospheric CPS ions (sp) are always seen at energies below the time-offlight cut-off energy (which is defined by $T=t_{s}-t_{o}$ and so depends on the pitch angle and the time elapsed since reconnection), whereas the injected sheath ions and energised magnetospheric ions are simultaneously present above this cut-off energy. This predicted continuation of $\mathrm{sp}$ ions at energies below the injected magnetosheath ions is a feature of all observations of dispersed LLBL and cusp ions, at both middle and low altitudes.

\section{Injection locations of observed cusp and LLBL ions}

It is instructive to return to the debate about where the precipitating ions seen in the cusp region were injected across the magnetopause. As was discussed in the introduction, Menietti and Burch (1988) used the ion Vs modelled in Fig. 7 to derive a spread of source locations of about $1 \mathrm{R}_{\mathrm{E}}$, whereas Lockwood and Smith (1993) argued that the spread of ion energies seen in low- 
altitude data reveal ions injected over regions $10-20 R_{E}$ wide. The modelling by Onsager et al. (1993) gave source extents which were somewhere between the two. Lockwood and Smith (1994) and Lockwood (1997) have discussed several reasons for these discrepancies. Menietti and Burch argued from the similarity of their estimate to the known dimensions of magnetopause flux transfer events (FTEs) that the cusp precipitation originated in bursts of reconnection; however, Lockwood and Smith (1994) point out that because the ions are largely frozen onto each newly opened field line, the distribution function seen on any one field line (and thus the width of the cusp ion V) depends on the sequence of ions injected onto that field line as a function of time, not on what happens to other field lines around it. Thus the precipitation is determined by the evolution of each newly opened field line after reconnection and is independent of the reconnection rate with which it was opened. Lockwood and Smith also show that the reconnection-rate variations, rather than restricting the width of the dispersion signature, cause one of a number of step-like features, as recently modelled in spectrogram format by Lockwood and Davis (1996).

Figure 8 shows the differential energy flux spectrum $J_{E}(E)$ at three $\left(t_{s}-t_{o}\right)$ for field-aligned ions $\left(a_{s}=0\right)$. From the preceding discussion, we class that for $\left(t_{s}-t_{o}\right)=200 \mathrm{~s}$ as LLBL, that for $\left(t_{s}-t_{o}\right)=300 \mathrm{~s}$ as in the cusp near the cusp/LLBL boundary and that for $\left(t_{s}-t_{o}\right)=500 \mathrm{~s}$ as also being in the cusp but near the cusp/mantle border. In all three cases, the time-of-flight cut-off can be clearly defined: below this cut-off, the remnant sp ions are seen. The dotted line shows one possible one-count level of the instrument $\left(J_{E}=10^{7}\right.$ $\left.\mathrm{cm}^{-2} \mathrm{~s}^{-1} \mathrm{sr}^{-1}\right)$, below which no ions would be detected. One can see that the width (energy range) of the

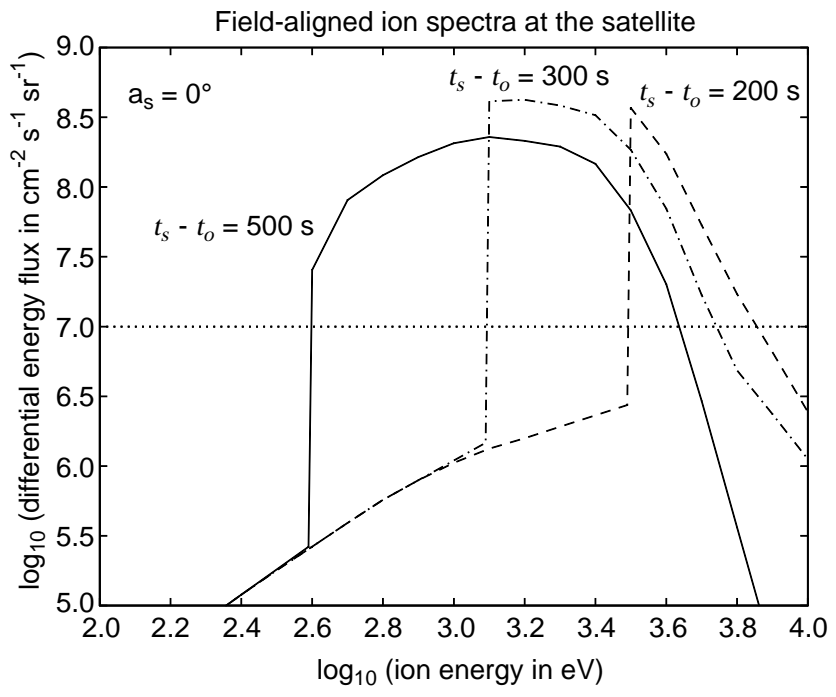

Fig. 8. Differential energy flux spectra of field-aligned ions $\left(a_{s}=0\right)$ detected at the mid-altitude satellite at three elapsed times since reconnection, $\left(t_{s}-t_{o}\right): 200 \mathrm{~s}$ (LLBL), $300 \mathrm{~s}$ (near the LLBL-cusp border) and $500 \mathrm{~s}$ (cusp). The dotted line shows an example threshold (imposed as a classification criterion or by the instrument one-count level) of $J_{E}=10^{7} \mathrm{~cm}^{-2} \mathrm{~s}^{-1} \mathrm{sr}^{-1}$ population detected depends on not only on $\left(t_{s}-t_{o}\right)$, but also on the one-count level: the width will decrease/ increase if the threshold $J_{E}$ is higher/lower. A corresponding decrease/increase in the energy width of the magnetosheath-like ion precipitation feature, as detected by any one instrument, would also be caused by a fall/rise in the solar-wind density, relative to that input into the model to generate the results shown here (see Table 1).

The velocity filter effect of the convection electric field means that a spread in ion energies at the satellite (at any one pitch angle) corresponds to a range of source locations (Lockwood and Smith, 1993). The range of detectable energies will depend on the instrument geometric factor, which sets the one-count level (note that because counts are proportional to differential energy flux $J_{E}$ for any one differential ion detector, the threshold (one-count level) $J_{E}$ is independent of energy). The spread of source locations of detected ions will increase with higher instrument sensitivity (lower onecount levels). This effect is investigated here in Fig. 9. The figure shows the distances $d_{n}$ from the reconnection site to the magnetopause source points $\left(\mathrm{P}_{\mathrm{n}}\right)$ of those ions in Fig. 8 whose flux equals the one-count level. These distances are given as a function of the value of that onecount level. For comparison, the dotted lines in Fig. 8 and 9 give one example of a threshold $J_{E}$ (one-count level) of $10^{7} \mathrm{~cm}^{-2} \mathrm{~s}^{-1} \mathrm{sr}^{-1}$. The three curves shown are for the same three values of $\left(t_{s}-t_{o}\right)$ as in Fig. 8 and are plotted using the same types of line. The curves in Fig. 9 mark the boundaries of the source regions of the detected ions and so define their apparent extent, as a function of the instrument one-count level.

If we consider the LLBL spectrum in Fig. 8 $\left(t_{s}-t_{o}=200 \mathrm{~s}\right)$, we see that the largest $J_{E}\left(E, a_{s}=0\right)$ is about $10^{8.5} \mathrm{~cm}^{-2} \mathrm{~s}^{-1} \mathrm{sr}^{-1}$ and that this is at the lower cut-off energy, $E_{i c}=2.93 \times 10^{3} \mathrm{eV}\left(\log _{10}\left(E_{i c}\right)=3.47\right)$.

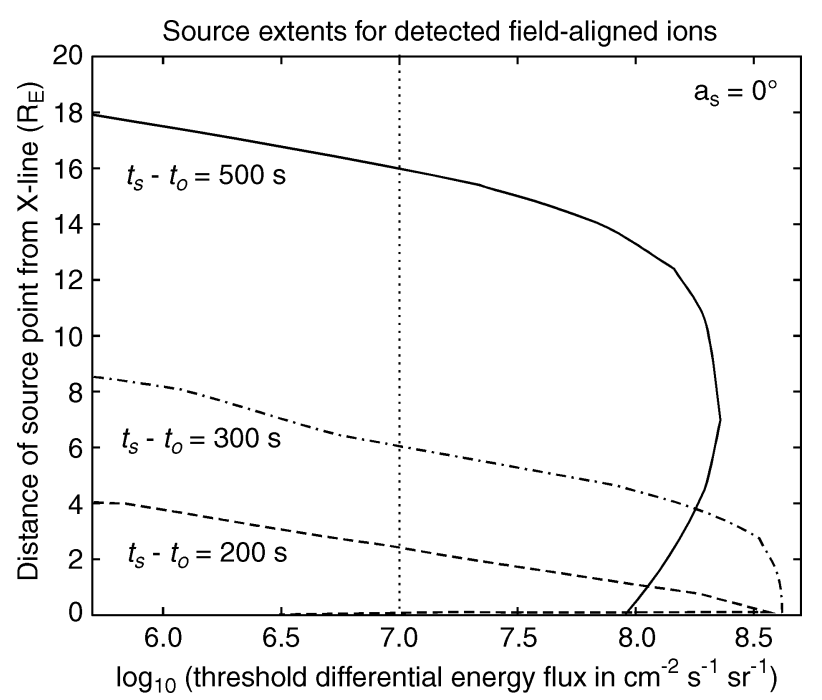

Fig. 9. The distance $d_{n}$ (of the magnetopause source point from the Xline) for ions whose flux $J_{E}$ is at a threshold level, as a function of that level and for the three elapsed times since reconnection $\left(t_{s}-t_{o}\right)$ used in Fig. 8 
These ions have the largest $T$ which, at a given $\left(t_{s}-t_{o}\right)$, means that they have the minimum $t_{n}$ [Eq. (3)] which is zero, i.e. these ions were injected at the reconnection site (at $d_{n}=0$ ). [As discussed by Lockwood $(1995 \mathrm{a}, \mathrm{b})$, there is a caveat which needs placing on this argument when the time-of-flight cut-off energy falls below the minimum energy injected at the X-line, which is $m V_{e x}{ }^{2} /$ 2 , where $V_{e x}$ is the value of $V_{e}$ in the immediate vicinity of the X-line; however, this only occurs at larger $\left(t_{s}-t_{o}\right)$ than in Fig. 8 and 9)]. As we lower the threshold in $J_{E}$, Fig. 8 shows that the low-energy limit of the detected ions does not change and these ions still all come from the X-line. However, ions are detected at increasingly higher energies and these ions thus have lower $T$ and higher $t_{n}$ and $d_{n}$. This can be seen in Fig. 9. For the lowest $J_{E}$ threshold shown $\left(10^{5.7} \mathrm{~cm}^{-2} \mathrm{~s}^{-1} \mathrm{sr}^{-1}\right)$, the upper $d_{n}$ has grown to $4 \mathrm{R}_{\mathrm{E}}$, i.e. the source region has expanded to cover a region on the low-latitude magnetopause $4 \mathrm{R}_{\mathrm{E}}$ wide from the reconnection site. At this $\left(t_{s}-t_{o}\right)$ of $200 \mathrm{~s}$, the field line that is being observed actually threads the boundary at a distance of $8.7 \mathrm{R}_{\mathrm{E}}$ from the X-line: thus if we could detect sheath ions of zero flight time (infinite energy) this would be the limit of the source region. This means that at this elapsed time since reconnection, the satellite is magnetically connected to the magnetic cusp region of the mid-latitude magnetopause (8.7 $\mathrm{R}_{\mathrm{E}}$ from the $\mathrm{X}$-line) but the precipitation at the satellite is classed as LLBL and was injected by flowing along open field lines across the lowlatitude magnetopause, within a few $R_{E}$ of the reconnection site.

For $\left(t_{s}-t_{o}\right)=300 \mathrm{~s}$, a very similar situation applies, but the source region is wider. The relatively flat top to the spectrum shown in Fig. 8 means that as we lower the threshold, the source region widens very rapidly after the first detection of the ions. This can be seen in Fig. 9, such that at $J_{E}$ of $10^{8.5} \mathrm{~cm}^{-2} \mathrm{~s}^{-1} \mathrm{sr}^{-1}$, the source region is $3 \mathrm{R}_{\mathrm{E}}$ wide, and this grows to over $8 \mathrm{R}_{\mathrm{E}}$ for $10^{5.7} \mathrm{~cm}^{-2} \mathrm{~s}^{-1}$ $\mathrm{sr}^{-1}$. At this $\left(t_{s}-t_{o}\right)$, the field line threads the boundary at $15.2 \mathrm{R}_{\mathrm{E}}$ from the $\mathrm{X}$-line.

However, for the case of $\left(t_{s}-t_{o}\right)=500 \mathrm{~s}$, the behaviour is significantly different. Fig. 8 shows that the peak $J_{E}\left(E, a_{s}=0\right)$ is not at the lower cut-off, but is at a higher $E$ of around $1 \mathrm{keV}$. Thus, as we lower the detection threshold, the first ions we see are not from the reconnection site in this case, rather they come from near $d_{n}=7 \mathrm{R}_{\mathrm{E}}$ (Fig. 9). The extent of this source region spreads rapidly about this $d_{n}$ until at a threshold $J_{E}$ of $10^{7.9} \mathrm{~cm}^{-2} \mathrm{~s}^{-1} \mathrm{sr}^{-1}$, the lower cut-off ions are seen for the first time: the source then extends from the X-line to $d_{n}$ of $14 \mathrm{R}_{\mathrm{E}}$. At the left of the plot, this source range has increased to $0-18 \mathrm{R}_{\mathrm{E}}$. These values are similar to those modelled by Lockwood (1995a) and are comparable to those inferred from observations by Lockwood and Smith (1993). At this $\left(t_{s}-t_{o}\right)$, the field line threads the boundary $39.5 \mathrm{R}_{\mathrm{E}}$. Notice, therefore, that the precipitation is classed as cusp on a field line that actually threads the magnetopause so far down the tail that it would be called part of the mantle.

The preceding discussion has been in terms of the effects of instrument sensitivity on the location and extent of the source of the ions seen. The same sort of effects will be present if the differential energy flux of the ions is used to define the precipitation classification. In other words, if one defines cusp as requiring flux values over a sufficiently high threshold, one will inevitably select those ions that come from a restricted region around the magnetic cusp.

\section{Summary and conclusions}

The open magnetosphere model can explain the energy/ pitch-angle dispersion of injected magnetosheath ions seen at middle and low altitudes, as well as the energy/ observation-time dispersion.

The ion distribution function evolves as a function of time elapsed since reconnection in a way that explains the BPS and LLBL precipitations as being on open field lines, in addition to the cusp, mantle and polar cap. The implications of this have been discussed by Lockwood (1997); specifically, some outstanding anomalies concerning the location of the precipitations with respect to the pattern of convection can be resolved.

The distribution seen in the LLBL is a mixture of magnetosheath ions and magnetospheric ions. However, at energies below the time-of-flight cut-off the magnetospheric ions are that part of the pre-existing closedfield-line (CPS) population which has not yet had time to be influenced by the fact that the field line has been opened. At energies above the magnetosheath-like ions, ions of magnetospheric origin are seen. However, these are not part of the same population as is seen simultaneously at lower energies. Instead, these ions have been reflected off the magnetopause. The reflection coefficient employed here has a constant value of 0.4 : this may be rather large, although it is consistent with the magnetopause observations of Fuselier et al. (1991). However, these ions would be detected even if this reflection is much weaker, because the ions are accelerated when they are reflected. The slope of the distribution function $f(E)$ is very steep at the hot tail of the distribution and thus the rise in energy $E$ tends to increase the phase space densities of the reflected ions: they can be detected at high energies even if the reflection coefficient is very low. There is, however, no reason why these two opposing effects should exactly counteract each other and make the re-sp tail an exact continuation of the remnant $\mathrm{sp}$ population. This can be seen to be true for many examples of boundary-layer spectra (e.g. Fig. 1 of Woch and Lundin, 1992).

The higher-energy, magnetosphere-like population which defines the LLBL (the re-sp) decays away as the field line evolves. This is because the ion acceleration turns to a deceleration as the field line straightens (plus, in reality, the reflection coefficient may decrease). In addition, the source sp population is progressively lost by interaction with the boundary. At the same time, the time-of-flight cut-off decreases with time elapsed since reconnection, allowing the lower-energy sheath ions to arrive. In this way the LLBL precipitation evolves into cusp as is often observed (e.g. Newell et al., 1991a; 
Woch and Lundin, 1992). Lockwood (1997) has pointed out that time-of-flight dispersion gives several severe conceptual difficulties if one thinks of the LLBL/cusp boundary as at the closed/open field line boundary.

The precipitation classed as LLBL at middle and low altitudes is on field lines that are magnetically connected to the mid-latitude magnetopause, i.e. which thread the magnetic cusp. Similarly, the precipitation classed as cusp is on field lines that thread the magnetopause in the high-latitude boundary layer (or mantle) and that classed as BPS is magnetically connected to the lowlatitude magnetopause. This is to be expected because the field lines evolve towards the tail during the flight time of the ions.

The extent of the source region of ions of a given classification depends on the threshold set to define that precipitation class and, in some cases, may be set by the instrument's sensitivity. A sufficiently high threshold definition of cusp particles restricts the source region to the vicinity of the magnetic cusp. However, a lower threshold shows that the particles in fact originate from the entire dayside magnetopause, at all latitudes down to the reconnection site. The threshold also influences the extent of the inferred source of LLBL ions. However, LLBL ions always arise from adjacent to the reconnection site.

Acknowledgements. The author is grateful to T. Onsager, S.W.H. Cowley and M.F. Smith for many discussions regarding this work. This work is supported by the UK Particle Physics and Astronomy Research Council.

Topical Editor D. Alcaydé thanks H.E. Petschek and another referee for their help in evaluating this paper.

\section{References}

Biernat, H. K., M. F. Heyn, R. P. Rijnbeek, V. S. Semenov and C. J. Farrugia, The structure of reconnection layers, application to the magnetopause, J. Geophys. Res., 84, 287-295 1989.

Burch J. L, P. H. Reiff, R. A. Heelis, J. D. Winningham, W. B. Hanson, C. Gurgiolo, J. D. Menietti, R. A. Hoffman, and J. N. Barfield, Plasma injection and transport in the mid-altitude polar cusp, Geophys. Res. Lett., 9, 921-924, 1982.

Burch, J. L., P. H. Reiff, J. D. Menietti, R. A. Heelis, W. B. Hanson, S. D Shawhan, E. G. Shelley, M. Suguira, D. R. Weimer, and J. D. Winningham, IMF $\mathrm{B}_{\mathrm{y}}$-dependent plasma flow and Birkeland currents in the dayside magnetosphere 1 . Dynamics explorer observations, J. Geophys. Res., 90, 15771593, 1985.

Burch, J. L., J. D. Menietti, and J. N. Barfield, DE-1 Observations of solar wind-magnetospheric coupling processes in the polar cusp, in Solar wind-magnetosphere coupling, Ed. Y. Kamide and J. A. Slavin, Terra Scientific Tokyo, pp. 441-452, 1986.

Chapman, S. C., and C. G. Moukis, A comparison of self-consistent kinetic and quasi-MHD simulations: application to a dipolarising field reversal, Geophys. Res. Lett., 23, 3251-3254, 1996.

Cowley, S. W. H., The causes of convection in the Earth's magnetosphere: a review of developments during IMS, Rev. Geophys., 20, 531-565, 1982.

Cowley, S. W. H., and C. J. Owen, A simple illustrative model of open flux tube motion over the dayside magnetopause, Planet. Space Sci., 37, 1461-1476, 1989.

Cowley, S. W. H., M. P. Freeman, M. Lockwood and M. F. Smith, The ionospheric signature of flux transfer events, in CLUSTER - dayside polar cusp, Ed. C. I. Barron, ESA SP-330, European
Space Agency Publications, Nordvijk, The Netherlands, pp 105-112, 1991.

de Hoffmann, F., and E. Teller, Magneto-hydrodynamic shocks, Phys. Rev., 80, 692-703, 1950.

Elliott, J. A., Plasma kinetic theory, in Plasma physics, Ed. by R. Dendy, Cambridge University Press, Cambridge, pp. 29-53, 1993.

Fuselier, S. A., D. M. Klumpar, and E. G. Shelley, Ion reflection and transmission during reconnection at the Earth's subsolar magnetopause, Geophys. Res. Lett., 18, 139-142, 1991.

Gosling, J. T., M. F. Thomsen, S. J. Bame, T. G. Onsager, and C. T. Russell, The electron edge of the low-latitude boundary layer during accelerated flow events, Geophys. Res. Lett., 17, 18331836, 1990.

Hill, T. W. and P. H. Reiff, Evidence of magnetospheric cusp proton acceleration by magnetic merging at the dayside magnetopause, J. Geophys. Res., 82, 3623-3628, 1977.

Kremser, G., J. Woch, K. Mursala, P. Tanskanen, B. Wilken, and R. Lundin, Origin of energetic ions in the polar cusp inferred from ion composition measurements by the Viking satellite, Ann. Geophyskae, 13, 595-607, 1995.

Lin, Y., and L. C. Lee, Structure of the dayside reconnection layer in resistive MHD and hybrid models, J. Geophys. Res., 98, 3919-3934, 1993.

Lockwood, M., The location and characteristics of the reconnection $\mathrm{X}$-line deduced from low-altitude satellite and ground-based observations: 1. theory, J. Geophys. Res., 100, 21791-21802, $1995 \mathrm{a}$.

Lockwood, M., Ground-based and satellite observations of the cusp: evidence for pulsed magnetopause reconnection, in Physics of the magnetopause, Ed. P.Song, B.U.O. Sonnerup, and M.F. Thomsen, American Geophysical Union Monograph 90, pp 417-426, 1995 b.

Lockwood, M., The relationship of dayside auroral precipitations to the open-closed separatrix and the pattern of convective flow, J. Geophys. Res., 102, 17475-17487, 1997.

Lockwood, M., T.G. Onsager, C.J. Davis, M.F. Smith and W.F. Denig,, The characteristics of the magnetopause reconnection $\mathrm{X}$-line deduced from low-altitude satellite observations of cusp ions, Geophys. Res. Lett., 2757-2760, 1994.

Lockwood, M., and C. J. Davis, On the longitudinal extent of magnetopause reconnection bursts, Ann Geophys., 14, 865-878, 1996.

Lockwood, M. and J. Moen, Ion populations on open field lines within the low-latitude boundary layer: theory and observations during a dayside transient event, Geophys. Res. Lett., 23, 28952898, 1996.

Lockwood, M., and M. F. Smith, Comment on "Mapping the dayside ionosphere to the magnetosphere according to particle precipitation characteristics" by Newell and Meng, Geophys. Res. Lett., 20, 1739-1740, 1993.

Lockwood, M., and M. F. Smith, Low- and mid-altitude cusp particle signatures for general magnetopause reconnection rate variations: I theory, J. Geophys. Res., 99, 8531-8555, 1994.

Lockwood, M., T. G. Onsager, C. J. Davis, M. F. Smith, and W. F Denig, The characteristics of the magnetopause reconnection Xline deduced from low-altitude satellite observations of cusp ions, Geophys. Res. Lett., 21, 2757-2760, 1994.

Lockwood, M., S. W. H. Cowley, and T. G. Onsager, Ion acceleration at both the interior and exterior Alfvén waves associated with the magnetopause reconnection site: signatures in cusp precipitation, J. Geophys. Res., 101, 21501-21515, 1996.

Menietti, J. D. and J. L. Burch, Spatial extent of the plasma injection region in the cusp-magnetosheath interface, J. Geophys. Res., 93, 105-114, 1988

Moen, J., D. Evans, H. C. Carlson and M. Lockwood, Dayside moving auroral transients related to LLBL dynamics, Geophys. Res. Lett., 23, 3247-3250, 1996

Newell, P. T., and C. -I. Meng, Mapping the dayside ionosphere to the magnetosphere according to particle precipitation characteristics, Geophys. Res. Lett., 19, 609-612, 1992. 
Newell, P. T. W. J. Burke, E. R. Sanchez, Ching-I. Meng, M. E. Greenspan, and C. R. Clauer, The low-latitude boundary and the boundary plasma sheet at low altitude: prenoon precipitation regions and convection reversal boundaries, J. Geophys. Res., 96, 21013-21023, 1991a.

Newell, P. T., W. J. Burke, C. -I. Meng, E. R. Sanchez and M. E. Greenspan, Identification an observation of the plasma mantle at low altitude, J. Geophys. Res., 96, 35-45, 1991b.

Onsager, T. G., C. A. Kletzing, J. B. Austin, and H. MacKiernan, Model of magnetosheath plasma in the magnetosphere: cusp and mantle precipitations at low altitudes, Geophys. Res. Lett., 20, 479-482, 1993.

Reiff, P. H., T. W. Hill, and J. L. Burch, Solar wind plasma injection at the dayside magnetospheric cusp, J. Geophys. Res., 82, 479-491, 1977.
Rosenbauer, H., H. Gruenwaldt, M. D. Montgomery, G. Paschmann, and N. Skopke, HEOS-2 plasma observations in the distant polar magnetosphere: the plasma mantle, J. Geophys. Res., 80, 2723-2737, 1975.

Smith, M. F. and Rodgers, D. J., Ion distributions at the dayside magnetopause, J. Geophys. Res., 95, 11617-11624, 1991.

Spreiter, J. R., A.L. Summers, and A. Y. Alksne, Hydromagnetic flow around the magnetosphere, Planet. Space Sci., 14, 223-253, 1966.

Woch, J., and R. Lundin, Magnetosheath plasma precipitation in the polar cusp and its control by the interplanetary magnetic field, J. Geophys. Res., 97, 1421-1430, 1992.

Woch, J., and R. Lundin, The low-latitude boundary layer at midaltitudes: identification based on Viking hot plasma data, Geophys. Res. Lett., 20, 979-982, 1993 